\title{
Aprendizaje Basado en Proyectos y Estrategias de Evaluación Formativas: Percepción de los Estudiantes Universitarios
}

\section{Project-based Learning and Formative Assessment Strategies: Perception of University Students}

\author{
Víctor Abella García * \\ Vanesa Ausín Villaverde \\ Vanesa Delgado Benito \\ Raquel Casado Muñoz \\ Universidad de Burgos, España
}

\begin{abstract}
En el ámbito de la Educación superior se están consolidando cambios metodológicos en los procesos de enseñanza aprendizaje. Estos cambios afectan a las estrategias didácticas, las cuales se están orientando hacia metodologías más activas. Pero también afectan a las estrategias de evaluación, buscando cada vez más la implicación del alumnado porque se ha mostrado como un elemento clave para mejorar el aprendizaje. En este artículo se presentan los resultados de una experiencia didáctica que combinó el Aprendizaje Basado en Proyectos (ABP) con estrategias de evaluación formativa. Una vez finalizada la experiencia, se realizó un estudio descriptivo mediante la aplicación de un cuestionario para analizar la percepción de los estudiantes sobre las estrategias de autoevaluación y evaluación entre iguales aplicadas durante todo el proyecto. Estuvieron implicados 120 estudiantes universitarios durante dos cursos académicos. Los resultados han mostrado que existen ciertas barreras que los estudiantes perciben cuando se implican en estrategias de evaluación formativa y compartida. Han manifestado no sentirse cómodos evaluando a sus compañeros, no pareciéndoles una experiencia muy motivadora; sin embargo, les parece un sistema más justo que si solo evalúa el profesor. Así mismo, destaca la importancia que para ellos tiene conocer desde el principio los criterios que van a guiar la evaluación, criterios que deben ser claros y comprensibles. Es clave continuar con la implantación y la investigación sobre estrategias de evaluación formativa y compartida que hagan consciente al estudiante tanto de su forma de aprender como de la necesidad de reflexionar sobre su trabajo desde una perspectiva crítica.
\end{abstract}

Palabras clave: Evaluación; Evaluación formativa; Aprendizaje activo; Educación superior; Autoevaluación.

\begin{abstract}
In the European Higher Education Area there are changes in the teaching and learning methodologies. These changes are affecting the didactic strategies, which are changing towards more active methodologies. But they also affect assessment strategies, increasingly seeking student involvement because it has been shown as a key element to improve learning. This paper shows the results of a didactic experience that combined Project Based Learning (PBL) with formative assessment strategies. Once the project was completed, a descriptive study was carried out through the application of a questionnaire to analyze the students' perception of the formative strategies of self-evaluation and peer-asessment applied during the development of the project. The experience involved 120 university students during two academic courses. The results have shown that there are certain barriers that students perceive when they are involved in formative and shared evaluation strategies. They indicated that they feel uncomfortable when they evaluate their classmates and they did not perceive the experience as motivating. However, the formative and shared evaluation has seemed to them a fairer system than only the teacher assesses their assignments. The results have also shown the importance of knowing from the beginning the criteria that will guide the evaluation, criteria that should be clear and understandable. It is important to continue with the implementation and research on formative and shared evaluation strategies that make the student aware of both their way of learning and the need to reflect on their work from a critical perspective.
\end{abstract}

Keywords: Assessment; Formative assessment; Active learning; Higher education; Selfassessment.

*Contacto: vabella@ubu.es

issn: $1989-0397$

www.rinace.net/riee/

https://revistas.uam.es/riee
Recibido: 1 de enero de 2020

$1^{\text {a }}$ Evaluación: 4 de febrero de 2020

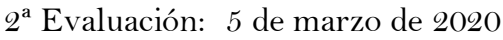

Aceptado: 18 de marzo de 2020 


\section{Introducción}

Son numerosos los cambios que ha supuesto el Espacio Europeo de Educación Superior en el actual sistema universitario, entre ellos los relativos a las metodologías de enseñanza-aprendizaje, incidiendo de manera especial en la forma de afrontar los procesos de evaluación. Se camina hacia la superación de enfoques centrados en el profesor y la clase magistral para ir incorporando metodologías más centradas en la actividad del estudiante (Lucena, 2016). Pero toda esta innovación no se debe centrar solo en la "forma de enseñar" sino que también debe llevar asociados cambios en la forma de evaluar. En este sentido, Buscá, Pintor, Martínez y Peire (2010) señalan que la evaluación tiene una gran importancia en el seguimiento y adquisición de competencias por parte del alumnado, por lo que se hace necesario también un enfoque cada vez más centrado en el propio alumno. Tradicionalmente ha estado relacionada con la realización de una única prueba final de carácter memorístico y que, por lo general, era la que determinaba la calificación final del estudiante (Palacios y López-Pastor, 2012). Sin embargo, la evaluación debe eludir la obtención de una única calificación final, tratando de asociar formación, seguimiento y aprendizaje como indicadores de la propia evaluación (Buscá et al., 2010; Carless, Joughin y Mok, 2006; Sanmartí, 2007).

La cuestión entonces es cómo integrar de manera conjunta esas nuevas estrategias de evaluación con metodologías docentes más centradas en el alumnado. En esta confluencia surge nuestra propuesta de combinar procesos de evaluación formativa con el Aprendizaje Basado en Proyectos (ABP) con el ánimo de dar un mayor protagonismo a los estudiantes. Consideramos que ambas estrategias deben ir unidas puesto que tal y como señala Arcos (2016), el ABP fomenta el espíritu autocrítico de los estudiantes, ya que son ellos quienes evalúan su propio trabajo y detectan los aspectos que deben ir mejorando.

\section{Fundamentación teórica}

Dentro de estas metodologías que buscan un mayor protagonismo por parte de los estudiantes encontramos el Aprendizaje Basado en Proyectos. Según nos indica el Buck Institute for Education (2013), la principal característica de esta metodología es que proporciona a los estudiantes un contexto real de aprendizaje y les implica de manera directa en el proceso de enseñanza aprendizaje. Esto se refleja en que será el estudiante el encargado de tomar una serie de decisiones encaminadas a resolver una tarea de cierto nivel de complejidad. De esta manera, para poder resolver con éxito esa tarea y realizar un adecuado proceso de toma de decisiones, la labor de investigación que lleven a cabo los estudiantes tendrá un papel fundamental (Grahame, 2011). En definitiva, son los estudiantes quienes planifican, deciden y elaboran el proyecto (Arcos, 2016).

De forma resumida podríamos decir que las principales características del ABP son las siguientes (Thomas, 2000):

- Se deben trabajar aspectos centrales del currículum y no centrar la propuesta en elementos periféricos del mismo.

- Una vez los estudiantes escogen el tema sobre el que trabajar las actividades deben conducir hacia contenidos esenciales de la temática. 
- Es fundamental que el proyecto o problemática planteada suponga un reto para los estudiantes. Es decir, el estudiante no debería poder resolver el proyecto planteado con los conocimientos que ya posee.

- Durante la realización del proyecto el estudiante debe progresar en cuanto a autonomía con el trabajo y en responsabilidad con la tarea. Esta progresión debe ser tanto a nivel cualitativo como a nivel cuantitativo.

- Un aspecto clave dentro del ABP es que plantee un desafío relacionado con la vida real.

En este sentido tal y como indican Ausín, Abella, Delgado y Hortigüela (2016), el ABP debe ser una herramienta para el desarrollo de competencias como la resolución de problemas, la creatividad, etc. que se deben potenciar en la actual configuración del Espacio Europeo de Educación Superior. Algunos estudios previos han mostrado que los alumnos que han estado inmersos en un ABP muestran una mejor capacidad para la resolución de problemas (Finkelstein, Hanson, Huang, Hirschman y Huang, 2010), mostrándose además más comprometidos con su aprendizaje y más autosuficientes (Thomas, 2000; Walker y Leary, 2009). Algunos estudios más recientes como los de Ausin y otros (2016) han mostrado que el ABP contribuye a fomentar la autonomía, la confianza en sí mismos y a incrementar la motivación. Estos mismos autores también indican que el ABP implica dejar de lado la enseñanza mecánica en favor de una metodología en el que las tareas se plantean como retos que siguen un hilo común y no como una asignación descontextualizada de diferentes tareas inconexas.

Desde el punto de vista de la evaluación durante las últimas décadas, tal y como hemos señalado con anterioridad, los estudiantes han sido evaluados mediante una única prueba, que por lo general solía ser un examen escrito. Además, el estudiante no solía contar con los criterios que se utilizaban para calificar esa prueba. Existen evidencias que muestran que el profesorado innova en cuanto a la práctica docente en el día a día (p. ej., AbellaGarcía, Delgado-Benito, Ausin-Villaverde y Hortigüela-Alcalá, 2019; González Losada y Triviño García, 2018; Hortigüela, Sánchez-Santamaría, Pérez-Pueyo y Abella, 2019), sin embargo, según indican Hortigüela Alcalá, Pérez-Pueyo y Abella García (2015) no muestran una incidencia directa en el necesario cambio respecto a los sistemas de evaluación empleados. De hecho, en la actualidad se sigue confundiendo el concepto de evaluación con el de calificación (Hortigüela, Pérez-Pueyo y González-Calvo, 2019). Siendo habitual en educación superior la aplicación de una continua calificación más que una evaluación continua. Esto ha provocado que encontremos que el profesorado utiliza dos sistemas de evaluación opuestos (López-Pastor y Palacios, 2012). Por un lado, el empleado por el profesorado que o bien no quiere o bien no sabe cómo afrontar ese cambio, y que estaría eminentemente orientado hacia la evaluación entendida como calificación. En el otro extremo se situaría una evaluación más orientada hacia el aprendizaje, en el que predominaría una evaluación formativa y continua. Este tipo de evaluación se ha mostrado como un elemento generador de aprendizaje (Ausín, Abella y Delgado, 2017; LópezPastor, Pintor, Muros y Webb, 2013), si bien su utilización no está tan extendida como podría ser deseable (Hortigüela et al., 2015).

Para conseguir un verdadero aprendizaje a partir de la evaluación es fundamental la implicación de los estudiantes en el proceso, puesto que mejora su nivel de implicación y motivación dentro del proceso de enseñanza aprendizaje general. La participación de 
profesores y estudiantes permite que se puedan poner en marcha estrategias de evaluación compartida, como son la autoevaluación, la coevaluación y la evaluación entre iguales (Gómez-Ruíz y Quesada-Serra, 2017), favoreciendo además la corresponsabilidad en el proceso de aprendizaje. Podemos entender la autoevaluación como la responsabilidad de los estudiantes para monitorizar y realizar juicios sobre su propio aprendizaje, puesto que a partir de unos criterios de evaluación explícitos el estudiante irá interiorizando los mismos a lo largo del proceso y ajustando sus respuestas a los objetivos de aprendizaje propuestos por el profesor. Además, este proceso permite a los estudiantes desarrollar unos hábitos de reflexión y de identificación de los propios errores, algo que les ayudará a ser conscientes de su propio aprendizaje y también a desarrollar su capacidad de aprender de forma autónoma (Delgado, Hortigüela, Ausín, Abella, 2018; Valero García y de Cerio, 2005). Sí resulta conveniente realizar un inciso en el concepto de coevaluación, ya que es un concepto que ha generado cierta confusión ya que se ha utilizado indistintamente para designar tanto la evaluación entre iguales (peer-assessment) como la evaluación compartida, colaborativa y cooperativa (Gómez-Ruíz y Quesada-Serra, 2017). En nuestro estudio vamos a aplicar la evaluación entre iguales (peer-assessment) más que una coevaluación (co-assessment). Realizamos esta aclaración porque la intención de nuestro trabajo es llevar a cabo un proceso de evaluación entre iguales con función formativa, buscando favorecer la autorreflexión y la mejora sobre los propios procesos de aprendizaje a partir de la valoración cualitativa de los estudiantes sobre el trabajo de sus compañeros (LopezPastor y Pérez-Pueyo, 2017). Algunos ejemplos de experiencias desarrolladas conjuntamente entre alumnos y profesores han puesto de manifiesto que la evaluación compartida mejora la calidad de los aprendizajes, a la vez que estimula la autorregulación y el desarrollo de competencias profesionales durante el proceso de evaluación (Delgado, Ausín, Hortigüela y Abella, 2016; Hortigüela, Abella, Delgado, Ausín, 2016; Hortigüela, Palacios y López-Pastor, 2019).

Por tanto, tal y como indican Fraile, Pardo y Panadero (2017), la utilización de estrategias de autoevaluación y coevaluación con una intención formativa, es decir de ayuda a la autorregulación y metacognición, consiste en la utilización de unos criterios de evaluación conocidos y aceptados por el alumno de antemano. De esta manera el estudiante podrá valorar de forma crítica tanto su progresión como la de otros compañeros, pudiendo obtener una retroalimentación que le va a permitir mejorar tanto en la realización de la tarea como en su comprensión del proceso de aprendizaje.

En definitiva, el objetivo de este artículo es conocer la percepción de los estudiantes sobre el proceso de evaluación formativa integrado dentro de un ABP. Concretamente trataremos de responder a las siguientes preguntas:

1. ¿Cómo se siente el estudiante en el proceso de evaluación entre iguales?

2. ¿Resultan motivadoras para el estudiante las estrategias de autoevaluación y evaluación entre iguales?

3. ¿Cómo perciben las estrategias de autoevaluación y evaluación entre iguales frente a otros sistemas?

4. ¿Cómo valoran conocer los criterios de evaluación desde el inicio de la asignatura? 


\section{Método}

\subsection{Participantes}

La muestra corresponde a los estudiantes que participaron en una experiencia didáctica, que se describirá más abajo, dentro de la asignatura TIC aplicadas a la Educación en el primer curso del Grado en Pedagogía durante los cursos 2016-2017 (42,5\%) y 2017-2018 $(57,5 \%)$. El total de la muestra lo forman 120 estudiantes, un $94,2 \%$ (113) mujeres frente un $5,8 \%(7)$ de hombres.

El rango de edades de los estudiantes estaba entre los 18 y los 25 años, siendo su edad media 19,15 años.

\subsection{Experiencia didáctica}

La experiencia educativa presentada, proyecto \#RadioEdUBU, se ha desarrollado en la asignatura "TIC aplicadas a la Educación" del Grado en Pedagogía de la Universidad de Burgos. Se trata de una asignatura de formación básica, impartida en primer curso y con una carga total de 6 créditos ECTS. Para su parte práctica se diseñó un proyecto que consiste en la creación por parte de los estudiantes de una radio educativa colaborativa a la que se ha denominado \#RadioEdUBU (Radio Educativa de la Universidad de Burgos). Esta radio educativa se nutre de podcast (episodios de radio) elaborados por los estudiantes de las titulaciones a través de la utilización de aplicaciones 2.0.

Como ya hemos señalado, se trabajó desde la metodología del Aprendizaje Basado en Proyectos. El proyecto se desarrolló en grupos de trabajo formados por 4 o 5 estudiantes, quienes funcionaron de forma autónoma en el marco de cada uno de sus grupos, explorando y analizando la información para alcanzar cada uno de los objetivos propuestos. Durante el transcurso del proyecto, cada grupo realizó un blog como porfolio del proyecto donde relataban sus avances y mostraban evidencias de los mismos: imágenes, documentos, enlaces, vídeos, etc. Para realizar el blog grupal se utilizó Blogger ya que se trata de una herramienta 2.0 gratuita y de fácil utilización.

Así mismo, a medida que avanzaba el proyecto, los grupos de trabajo debían realizar unos entregables que les servían como fecha de referencia para los avances dentro del proyecto. De esta forma, ninguno de los grupos se quedaba descolgado y garantizábamos un trabajo continuado. El proyecto se organizó en 5 fases:

1. Selección del tema. La elección del tema por parte de los estudiantes era libre, pero la temática debía estar relacionada con las TIC y la Educación. Además, debían justificar el interés y la relevancia del tema para el proyecto.

2. Entrevista radiofónica. Esta fase se dividió a su vez en:

a. Búsqueda y selección del experto a entrevistar. Se solicitó a los estudiantes que escogieran una persona experta en el tema elegido para realizar la entrevista radiofónica.

b. Guion inicial de la entrevista.

c. Grabación de la entrevista. Se dio total libertad a los estudiantes para que realizasen la entrevista con la herramienta que fuera más sencilla para ellos. De esta forma, exploraban diferentes posibilidades y escogían la aplicación que más se ajustase a sus necesidades. 
d. El último paso fue el apartado de edición y montaje de la entrevista. Se recomendó a los estudiantes que utilizasen Audacity, por ser un editor de audio de software libre. Una vez tuvieran la entrevista editada y montada deberían subirla a iVoox.

3. Vídeo promocional. Se pidió a los estudiantes que crearan un vídeo promocional de la entrevista utilizando la técnica de animación Cut out. Antes de crear el vídeo se les pidió también que realizarán un guion gráfico o storyboard (Entregable 4) del mismo. Tras realizar el vídeo, cada uno de los grupos subió a YouTube el archivo de vídeo.

4. Google Sites. Consistió en la elaboración de un sitio web para presentar el trabajo final. Esta página web tenía una estructura común para todos los grupos y debía recoger todos los elementos diseñados durante el proyecto.

5. Presentación oral y defensa. Los estudiantes defendieron cómo habían desarrollado el proyecto, los pasos que dieron, los problemas a las que tuvieron que hacer frente y cómo los habían solucionado. Además, realizaron una valoración de la entrevista, expusieron las ideas principales del tema elegido y, finalmente, reflexionaron sobre el propio proyecto.

Tal y como hemos recogido en la introducción, se ha optado por un proceso de autoevaluación y evaluación entre iguales con intención formativa y compartida. Al inicio del curso se proporcionó a los estudiantes los criterios de evaluación del portafolio electrónico (blog) y del proyecto mediante rúbricas de evaluación. A lo largo del proyecto se realizaron distintos procesos de autoevaluación y evaluación entre iguales del trabajo que se iba desarrollando. Estos procesos se basaban en los criterios de evaluación reflejados al inicio de la asignatura. De esta manera los estudiantes autoevaluaban en grupo el trabajo que habían desarrollado hasta el momento tanto en el blog como en el proyecto. Para la evaluación entre iguales cada grupo de trabajo evaluó a otro que se le asignó de forma aleatoria. Con el objetivo de que estos procesos mejoraran la autorreflexión y la percepción sobre el propio trabajo cada grupo "evaluador" debía explicar y justificar al grupo "evaluado" el resultado de su evaluación, contrastándolo además con su propia autoevaluación.

\subsection{Instrumentos}

Con la intención de dar respuesta a las preguntas de investigación previamente realizadas, se elaboró un cuestionario ad hoc con 8 ítems. A cada uno de los ítems se respondía mediante una escala tipo Likert de 1 (nada de acuerdo) a 5 (totalmente de acuerdo).

1. Me he sentido cómodo evaluando a otros compañeros.

2. Me he sentido cómodo sabiendo que otros compañeros me estaban evaluando.

3. Evaluar a otros compañeros me ha parecido motivador.

4. Considero que la autoevaluación y la evaluación entre iguales mediante rúbricas es un sistema de evaluación justo.

5. Considero que con los procesos de autoevaluación y evaluación entre iguales la calificación es más justa que si solo evalúa el profesor.

6. Conocer los criterios de evaluación desde el principio hace que sea más eficaz en el desarrollo del trabajo 
7. Conocer los criterios de evaluación desde el inicio del trabajo hace que tenga menos dudas sobre el trabajo que tengo que realizar.

8. Conocer los criterios de evaluación desde el inicio del trabajo es más justo que otros procesos de evaluación en el que no se conocen los criterios.

\subsection{Procedimiento}

El estudio cumplió con los valores y prácticas éticas requeridas dentro de la investigación educativa: consentimiento voluntario informado, derecho a la información, protección de datos y garantías de confidencialidad, anonimato y no discriminación.

Una semana antes del final del curso el cuestionario fue entregado en persona a los estudiantes. Se administró durante las clases prácticas obligatorias con el objetivo de obtener el número máximo de respuesta. No obstante, con la intención de que no hubiera percepciones de coerción en los estudiantes la cumplimentación del cuestionario fue voluntaria (Roberts y Allen, 2015).

\subsection{Análisis de datos}

Se realizaron análisis descriptivos de análisis de medias y desviaciones típicas, seguidos de análisis de distribución de frecuencias con la intención de conocer el número de estudiantes que se agrupaban en cada categoría dentro las preguntas realizadas.

\section{Resultados}

Cuadro 1. Medias y desviaciones típicas de los ítems analizados

\begin{tabular}{|c|c|c|}
\hline ÍTEM & MEDIA & $\begin{array}{l}\text { DESVIACIÓN } \\
\text { TÍPICA }\end{array}$ \\
\hline Me he sentido cómodo evaluando a otros compañeros. & 3,18 & 1,07 \\
\hline $\begin{array}{l}\text { Me he sentido cómodo sabiendo que otros compañeros me estaban } \\
\text { evaluando. }\end{array}$ & 3,34 & 1,01 \\
\hline Evaluar a otros compañeros me ha parecido motivador. & 3,12 & 0,96 \\
\hline $\begin{array}{l}\text { Considero que la autoevaluación y la evaluación entre iguales } \\
\text { mediante rúbricas es un sistema de evaluación justo. }\end{array}$ & 3,83 & 0,88 \\
\hline $\begin{array}{l}\text { Considero que con los procesos de autoevaluación y evaluación entre } \\
\text { iguales la calificación es más justa que si solo evalúa el profesor. }\end{array}$ & 3,68 & 1,04 \\
\hline $\begin{array}{l}\text { Conocer los criterios de evaluación desde el principio hace que sea más } \\
\text { eficaz en el desarrollo del trabajo }\end{array}$ & 4,22 & 0,68 \\
\hline $\begin{array}{l}\text { Conocer los criterios de evaluación desde el inicio del trabajo hace que } \\
\text { tenga menos dudas sobre el trabajo que tengo que realizar. }\end{array}$ & 4,08 & 0,87 \\
\hline $\begin{array}{l}\text { Conocer los criterios de evaluación desde el inicio del trabajo es más } \\
\text { justo que otros procesos de evaluación en el que no se conocen los } \\
\text { criterios. }\end{array}$ & 3,98 & 0,75 \\
\hline
\end{tabular}

Fuente: Elaboración propia.

En el cuadro 1 se presentan las Medias y Desviaciones Típicas de los ítems del cuestionario, siendo el rango de respuestas de 1 a 5 en todos los casos. Tal y como se puede apreciar los tres ítems con mayor puntuación media, y menor desviación típica, son los relacionados con el conocimiento de los criterios de evaluación desde el inicio de la asignatura. El ítem que mayor media obtiene $(M=4,22)$ es el que relaciona el conocimiento de los criterios de evaluación con la eficacia en el desarrollo del trabajo, siendo también el que menor dispersión de los datos presenta $(D T=0.68)$. Siguiendo en orden descendente en el valor de la media están los ítems que se relacionan con la 
percepción de los estudiantes de las estrategias de evaluación formativa utilizadas frente a otros sistemas de evaluación. Es decir, cuando les preguntamos si el uso de rúbricas en estos procesos hace que la evaluación sea más justa $(\mathrm{M}=3,83$, DT $=0,88)$ y cuando preguntamos si consideran que la evaluación con estos sistemas es más justa que cuando evalúa solo el profesor $(\mathrm{M}=3,68, \mathrm{DT}=1,04)$. Con menor puntuación media aparecen los dos ítems relacionados con cómo se sienten los estudiantes durante el proceso de evaluación entre iguales. Es decir, si se siente cómodos cuando otros compañeros le evalúan $(\mathrm{M}=3,34, \mathrm{DT}=1,01)$ y si se sienten cómodos cuando tienen que ser ellos los que evalúan a otros compañeros $(\mathrm{M}=3,18 \mathrm{DT}=1,07)$. Finalmente, el ítem que menor puntuación media presenta $(\mathrm{M}=3,12, \mathrm{DT}=0,96)$ es el que cuestiona a los estudiantes si evaluar a otros compañeros les ha resultado motivador.

El primer aspecto a analizar en la distribución de frecuencias fue conocer cómo se sienten los estudiantes en el proceso de evaluación entre iguales. En primer lugar, se preguntó a los estudiantes si se sienten incómodos cuando son evaluados por otros estudiantes. Tal y como se puede observar en la figura 1 la mayor parte de ellos $(45,8 \%)$ se sienten cómodos sabiendo que están siendo evaluados por otros compañeros. En el polo opuesto tenemos que algo más de un cuarto de los estudiantes $(26,7 \%)$ indican que no se sienten cómodos cuando tienen que evaluar a otros compañeros, si bien es sólo el 2,5\% de los participantes los que se sienten muy incómodos. Es destacable también que el 32,5\% de los estudiantes se sitúan en una posición intermedia.

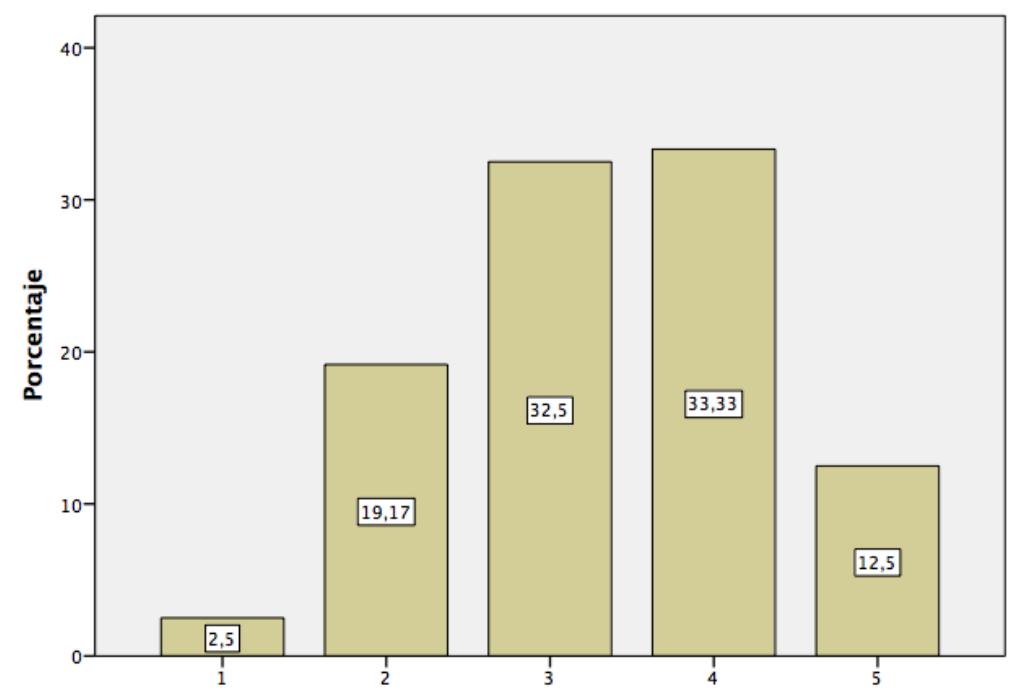

Figura 1. Distribución de frecuencias sobre si los estudiantes se han sentido cómodos siendo evaluados por otros compañeros

Fuente: Elaboración propia.

En cambio, cuando se pregunta a los estudiantes si se han sentido cómodos cuando tienen que evaluar a otros compañeros (figura 2) la mayor parte de los estudiantes $(39,16 \%)$ sí se sienten cómodos cuando otros compañeros son los encargados de evaluarles. En este caso un $34,17 \%$ de los estudiantes se muestran en una posición neutral, es decir que no se sienten cómodos, pero tampoco incómodos. Por su parte, el 26,66\% no se sienten cómodos siendo evaluados por otros compañeros. 


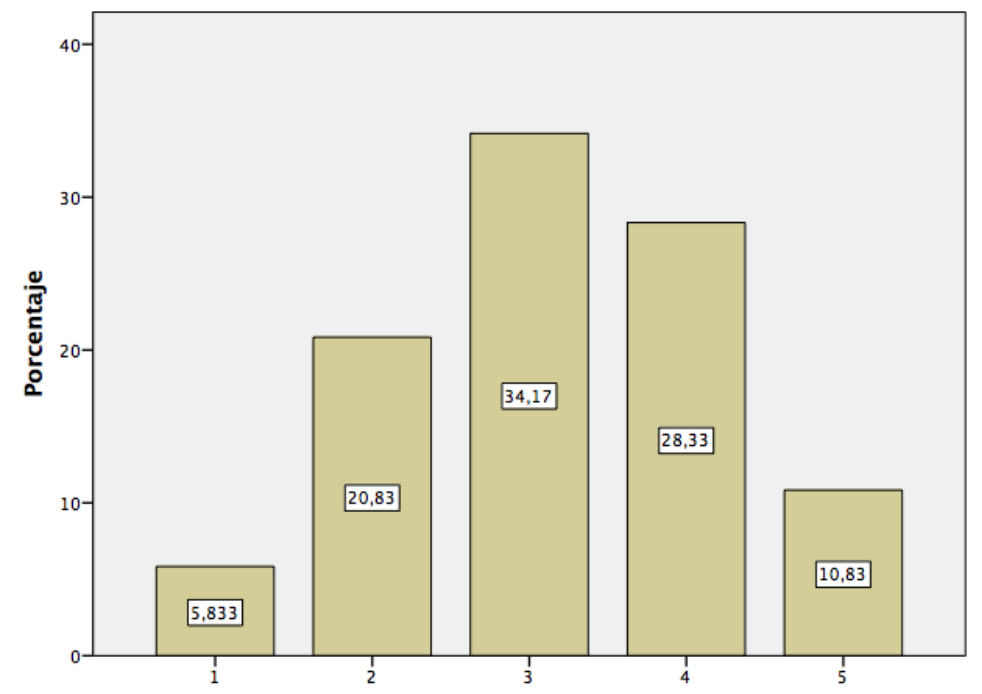

Figura 2. Distribución de frecuencias sobre cómo se han sentido los estudiantes cuando tienen que evaluar sus compañeros

Fuente: Elaboración propia.

El segundo aspecto a evaluar se centraba en si les ha resultado motivador evaluar a otros compañeros (figura 3). En este caso los resultados han mostrado que casi la mitad de los estudiantes, $49 \%$, han escogido la opción de respuesta neutra. Es decir que el proceso de evaluación entre iguales no les ha parecido motivador, pero tampoco desmotivador. A algo más de un $21 \%$ les ha parecido que la experiencia de evaluar a otros compañeros no ha sido motivadora. Finalmente, un 30\% de los participantes en la experiencia sí considera que evaluar a otros compañeros es algo que les ha resultado motivador.

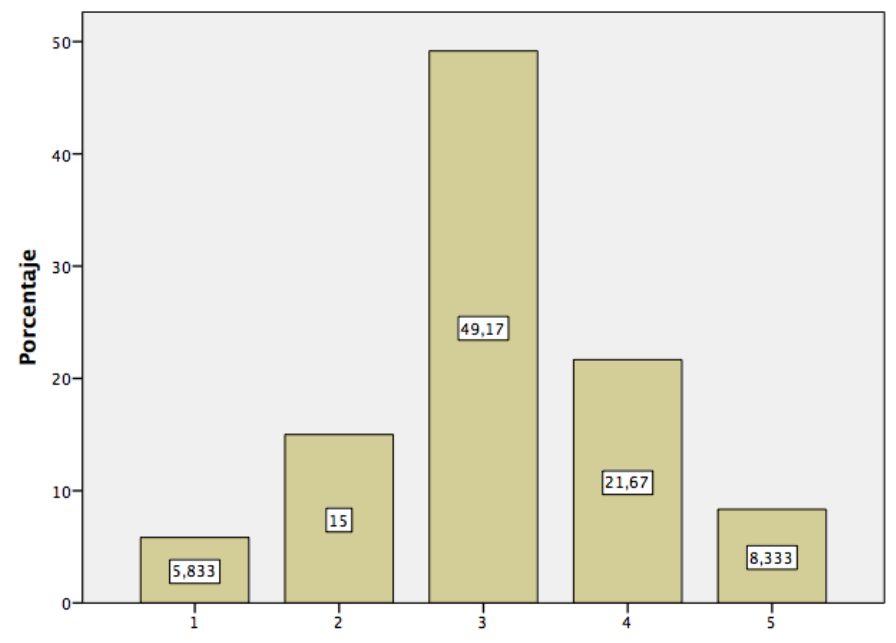

Figura 3. Distribución de frecuencias sobre la motivación de los estudiantes en la evaluación entre pares

Fuente: Elaboración propia.

Otra cuestión a analizar, siguiendo nuestras preguntas de investigación, es cómo perciben las estrategias de autoevaluación y evaluación entre iguales frente a otros sistemas. En primer lugar, se preguntó si consideraban que la autoevaluación y la evaluación entre 
pares mediante rúbricas era un sistema de evaluación justo. Ninguno de los participantes en el proyecto se mostró en total desacuerdo con la afirmación (figura 4). De esta manera un $6,66 \%$ sí le parece que el sistema de evaluación formativa aplicado mediante rúbricas no les parece un sistema de evaluación justo. Yendo al polo positivo nos encontramos con que un $65 \%$ de los estudiantes lo considera un sistema justo o muy justo. De nuevo nos encontramos con un porcentaje importante, más del $28 \%$, se sitúa en una posición neutral.

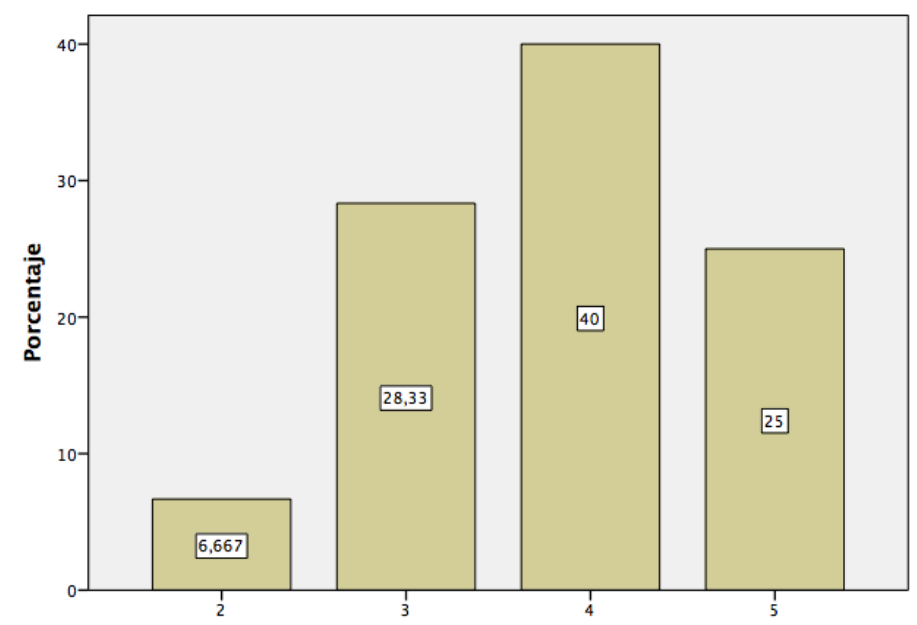

Figura 4. Distribución de frecuencias sobre si consideran que la autoevaluación y la evaluación entre pares mediante rúbricas es un sistema de evaluación justo Fuente: Elaboración propia.

A la afirmación "Considero que con los procesos de autoevaluación y evaluación entre iguales la calificación es más justa que si solo evalúa el profesor" la mayor parte de las respuestas obtenidas han sido positivas (figura 5). Concretamente un $60 \%$ está de acuerdo o muy de acuerdo con que se obtiene una calificación final más justa que si únicamente es el profesor el que lleva a cabo la evaluación. Siguiendo en orden descendente un 27,5\% escoge la posición neutral. Finalmente, en un porcentaje algo superior al $12 \%$ se encuentran los estudiantes que consideran que es más justa la evaluación realizada únicamente por el profesor frente a las estrategias de autoevaluación y evaluación entre iguales, si bien son tan solo un 3,33\% los que se manifiestan totalmente en contra.

También se preguntó sobre su percepción acerca de conocer los criterios de evaluación desde el principio de la asignatura. La primera pregunta relacionada con esta cuestión hacía referencia a si tener presentes los criterios desde el inicio de la asignatura hace que sea más eficaz en el desarrollo del trabajo por su parte (figura 6). En este caso los resultados obtenidos son muy positivos, puesto que el $87,5 \%$ ha respondido de forma favorable, es decir que saber de antemano cómo y con qué criterios van a ser evaluados hace que se centren en los aspectos relevantes de la tarea. Por el contrario, tan solo un 0,83\% consideran que no les ha hecho ser más eficaces en su tarea. En este caso los que se sitúan en una posición neutral no alcanzan el 12\%. 


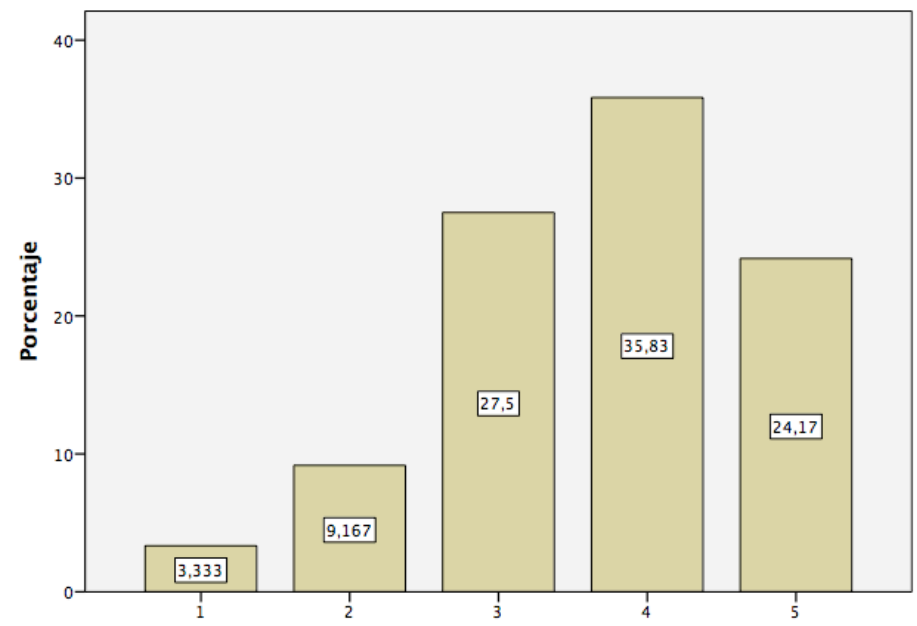

Figura 5. Distribución de frecuencias sobre si en los procesos de autoevaluación y evaluación entre pares la calificación es más justa que si solo evalúa el profesor Fuente: Elaboración propia.

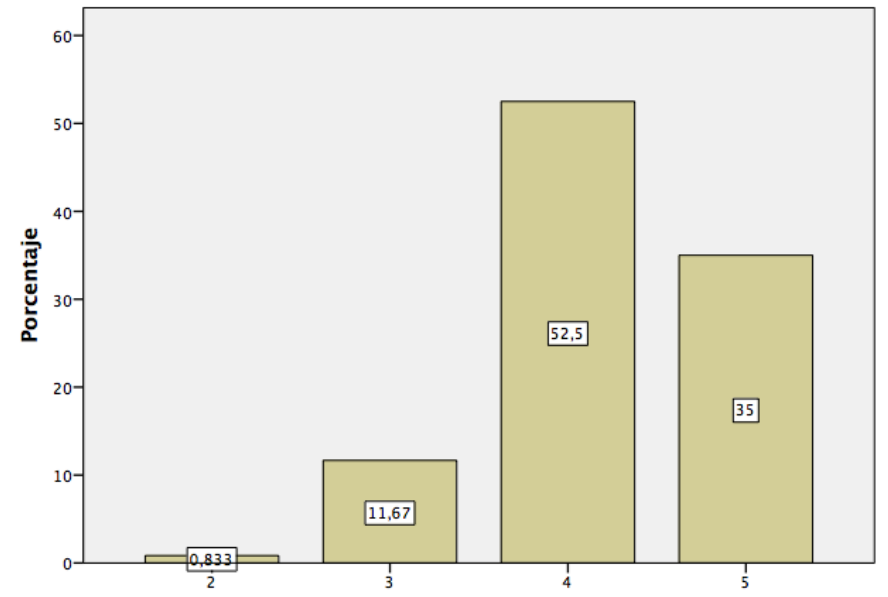

Figura 6. Distribución de frecuencias sobre la influencia de los criterios de evaluación fijados y su eficacia en el desarrollo del trabajo

Fuente: Elaboración propia.

Siguiendo con las cuestiones relacionadas con el conocimiento de los criterios de evaluación desde el inicio de la asignatura, también se les preguntó si les ayudaba a la hora de realizar el proyecto, concretamente si les hacía tener menos dudas sobre el trabajo que tenían que realizar (figura 7). De nuevo los resultados han vuelto a ser muy positivos ya que el $78,3 \%$ de los estudiantes sí cree que estos criterios les orientan en el trabajo que deben ir desarrollando a lo largo del proyecto. En este caso el porcentaje de alumnos que se sitúan en posiciones neutrales es de un 18,33\%. Nuevamente el porcentaje de estudiantes que considera que conocer los criterios de antemano no les ha ayudado a tener menos dudas vuelve a ser muy bajo, apenas superando el $3 \%$. 


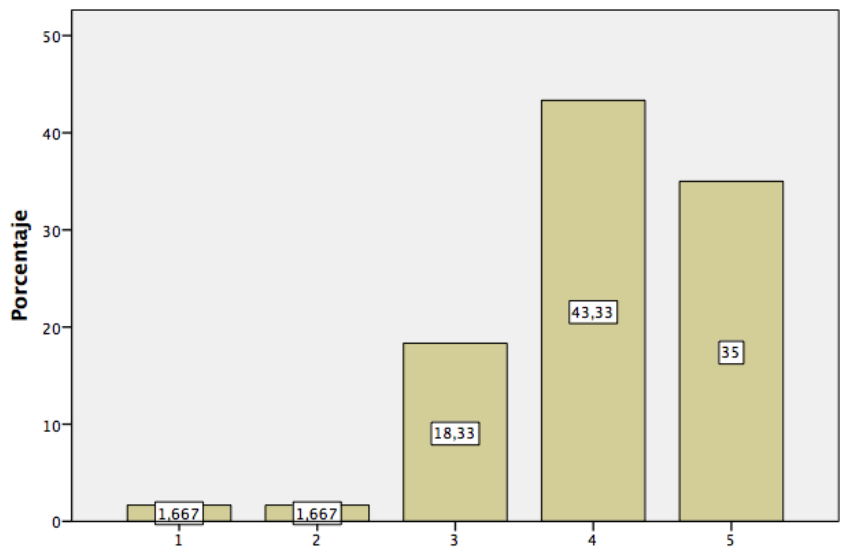

Figura 7. Distribución de frecuencias sobre si conocer los criterios de evaluación indicados en la rúbrica desde el inicio del trabajo hace que el estudiante tenga menos dudas sobre el trabajo que tiene que realizar Fuente: Elaboración propia.

Finalmente se preguntó si conocer los criterios de evaluación desde el inicio del proyecto les ha parecido más justo frente a otros procesos de evaluación en los que no se conocen los criterios con los que sus trabajos van a ser evaluados (figura 8). Tras el desarrollo del proyecto basado en ABP y la aplicación de distintas estrategias de evaluación formativa y compartida, el 73,33\% de los estudiantes considera que el conocer los criterios desde el inicio les ha parecido un sistema de evaluación más justo frente a otras actividades de evaluación en la que no han tenido ese conocimiento previo. En el polo opuesto un 0,83\% ha considerado que el sistema aplicado no ha sido más justo, mientras que casi un $26 \%$ han optado por la respuesta que no muestra ni acuerdo ni desacuerdo con la afirmación planteada.

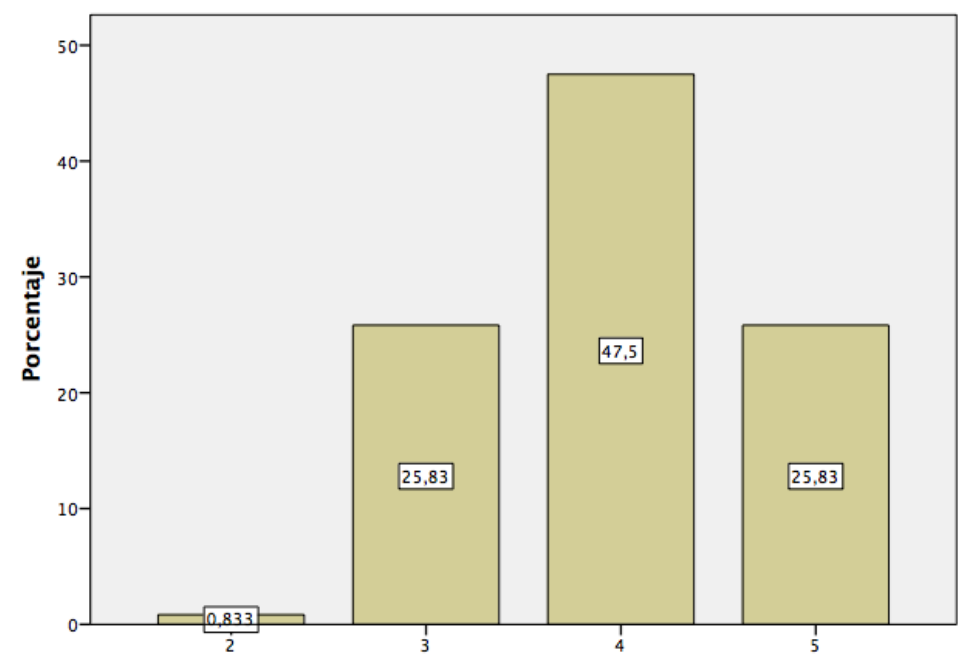

Figura 8. Distribución de frecuencias sobre si conocer los criterios de evaluación desde el inicio del trabajo es más justo que otros procesos de evaluación Fuente: Elaboración propia. 


\section{Discusión y conclusiones}

El objetivo de este artículo ha sido analizar cómo perciben los estudiantes un proceso de evaluación formativa, centrado en estrategias de autoevaluación y evaluación entre iguales, dentro de una metodología de Aprendizaje Basada en Proyectos. Este objetivo se ha articulado en torno a cuatro preguntas de investigación, las cuales utilizaremos como guía para el desarrollo de este apartado.

Tal y como indicaban Hortigüela y colaboradores (2015), los procesos de evaluación formativa no se encuentran tan extendidos como se desearía. Por eso cuando enfrentamos a los estudiantes a estrategias de autoevaluación y evaluación entre iguales debemos pensar que son estrategias a las que se enfrentan en muchos casos por primera vez. A partir de los resultados obtenidos vemos que existe cierta desconfianza de los estudiantes hacia este tipo de estrategias. Indican que no se acaban de sentir cómodos cuando tienen que evaluar a otros compañeros. La explicación que se puede dar es que, como se ha indicado, son prácticas poco extendidas y, en muchos casos, aunque se hayan aplicado se han hecho con un carácter sumativo o de obtención de una calificación final. La aplicación de la autoevaluación, la evaluación entre iguales y la coevaluación desde un punto de vista sumativo supone orientar la evaluación hacia la calificación más que hacia el aprendizaje (López Pastor y Palacios, 2012), impidiendo la toma de conciencia del propio proceso de aprendizaje y la propia autorregulación (Panadero y Alonso-Tapia, 2013). Por otro lado, debemos pensar que en la mayoría de las experiencias previas de los estudiantes la responsabilidad de la evaluación ha sido una tarea exclusiva del profesor, algo también de hondo calado a nivel social. Se considera que una de las labores del profesorado es corregir para otorgar una calificación final en función de lo aprendido. Es por eso por lo que tal vez los estudiantes perciban que están haciendo la labor del profesor más que contando con una oportunidad para mejorar su propio proceso de aprendizaje.

Esa idea de que los alumnos piensan que están haciendo una labor que no les corresponde a ellos y sí al profesor, podría ayudar a explicar los resultados obtenidos en el plano de la motivación. Hemos visto que el aplicar estrategias de evaluación entre iguales no les ha resultado muy motivador, dentro de las estrategias de evaluación formativa ya que, desde nuestro punto de vista, los estudiantes siguen estando muy orientados hacia la calificación final. Sería importante analizar cómo interpretan las estrategias en las que no se les otorga una calificación numérica de su trabajo y sí una evaluación más cualitativa.

Por otro lado, también nos hemos cuestionado cómo perciben los sistemas de autoevaluación y evaluación entre pares que hemos utilizado y, además, cómo los valoran frente a otras estrategias de evaluación sumativa. Hemos observado que un porcentaje no muy elevado de los estudiantes considera que la utilización de rúbricas dentro de las estrategias de evaluación formativa que hemos utilizado no le parece un sistema de evaluación justo. No obstante, debemos tener en cuenta que es un porcentaje mucho menor que aquellos que no se sienten cómodos evaluando a otros compañeros o siendo evaluados por ellos. Sí es destacable el elevado porcentaje de alumnos que se sitúa en posiciones neutrales, es decir sobre los que la experiencia no ha tenido un impacto ni negativo ni positivo. Una posible solución puede ser la de negociar con ellos los criterios de evaluación con el objetivo de que los interioricen y muestren una mayor confianza en los criterios establecidos (Braund y de DeLuca, 2018). A pesar de esto, los estudiantes siguen mostrando cierta desconfianza hacia que la calificación sea otorgada únicamente por el profesor. En este sentido resulta llamativo que el proceso de evaluación formativo no haya 
resultado muy motivador en general y sin embargo en su mayoría crean que es un sistema de evaluación más justo que el realizado solo por el profesor. Una posible explicación es que en el sistema de evaluación que hemos aplicado, además de ser formativo, los criterios de evaluación y calificación se han hecho explícitos desde el primer día. Sin embargo, casi con total seguridad, sus experiencias previas se relacionen con estrategias en las que el profesor es el encargado de poner una calificación sin indicar los criterios de evaluación utilizados. Sería interesante analizar en estudios posteriores qué experiencias previas han tenido los estudiantes respecto a la evaluación, para ver cómo influyen estas experiencias en las respuestas. Debemos tener en cuenta que las estrategias de autoevaluación, evaluación entre pares y coevaluación no están muy extendidas y, en muchas ocasiones, se utilizan más desde el punto de vista sumativo que formativo.

Finalmente, nos preguntábamos cómo valoran los estudiantes conocer los criterios de evaluación desde el inicio de la asignatura. A este respecto es necesario aclarar que si hay algo en lo que coinciden los autores es que, para aplicar la autoevaluación, la evaluación entre pares y la coevaluación con una función verdaderamente formativa, el alumnado debe conocer los criterios de evaluación, criterios que además deben ser claros y comprensibles para los estudiantes (Durán, 2014; López-Pastor y Pérez-Pueyo, 2017). Es decir que no basta con hacer explícitos los criterios, sino que además el éxito de la aplicación de las estrategias de autoevaluación, coevaluación y evaluación entre pares va a depender de su interiorización por parte de los estudiantes (Braund y DeLuca, 2018). En este apartado es dónde hemos obtenido los mejores resultados de nuestra experiencia. Se ha puesto de manifiesto que proporcionar unos criterios comprensibles con antelación evita la incertidumbre y desde el primer momento pueden orientar la tarea hacia metas concretas. No obstante, los criterios de evaluación no dejan de ser los que determinan la calidad de un trabajo en función de unos niveles de referencia (Fraile et al., 2017).

En definitiva, hemos visto cómo los estudiantes mantienen algunas reticencias cuando se aplican estrategias de evaluación formativa, tal y como ya reflejaron Hortigüela y otros (2015). Encontrarnos que estas reticencias no es algo extraño, sino más bien algo habitual cuando se inician estas prácticas ya que los estudiantes se tienen que acostumbrar a cambiar su enfoque de estudio y aprendizaje (Martínez, Castejón y Santos, 2012). Sin embargo, insistimos en que sí han percibido como muy positivo el conocer desde el primer momento los criterios de evaluación que se iban a seguir a lo largo de la asignatura, tanto a nivel de orientación de las tareas a realizar como desde el punto de vista del ajuste de la evaluación y los resultados obtenidos a la realidad.

Por otro lado, debemos interpretar estos resultados con cierta cautela ya que el estudio presenta ciertas limitaciones. En primer lugar, los resultados se basan en el análisis de un proyecto concreto que se realiza en una única materia de una sola titulación. Tampoco debemos olvidar que la muestra presenta un sesgo hacia el sexo femenino que, si bien en las titulaciones relacionadas con el ámbito de la educación la presencia femenina suele ser más elevada, es una limitación a tener en cuenta. Por estas razones se hace necesario seguir indagando, desde enfoques cualitativos, los puntos de unión entre el ABP y las estrategias de evaluación formativa con mayor diversidad metodológica y de estudiantes. Dichos enfoques cualitativos nos permitirán confrontar además los puntos de vista tanto de docentes como de estudiantes, centrándonos en el impacto que sobre los estudiantes tiene la retroalimentación recibida a lo largo del proceso tanto desde el punto de vista del profesor como desde el punto de vista de los compañeros. Tampoco debemos dejar de lado el impacto que estas estrategias tienen sobre el aprendizaje adquirido por los estudiantes, 
así como la transferencia a otras asignaturas y contextos del conocimiento adquirido sobre cómo aprenden y sus estrategias de reflexión y autoevaluación del trabajo realizado.

\section{Referencias}

Abella-García, V., Delgado-Benito, V., Ausin-Villaverde, V. y Hortigüela-Alcalá (2019). To tweet or not to tweet: Student perceptions of the use of Twitter on an undergraduate degree course. Innovations in Education and Teaching International, 56(4), 402-4011. https://doi.org/10.1080/14703297.2018.1444503

Arcos, A. (2016). Aprendizaje Basado en Proyectos (ABP). ENIAC, Espacio de pensamiento $e$ innovación educativa. Recuperado de https://issuu.com/gruposiena/docs/12097suplemento_eniac?e=8701546/35507538

Ausín, V., Abella, V. y Delgado, V. (2017). Implicación del alumnado en la evaluación a través del portafolio electrónico. Infancia, Educación y Aprendizaje 3(2), 77-81.

Ausín, V., Abella, V., Delgado, V. y Hortigüela, D. (2016). Aprendizaje Basado en Proyectos a través de las TIC. Una experiencia de innovación docente desde las aulas universitarias. Formación Universitaria, 9(3), 31-38. https://doi.org/10.4067/S0718-50062016000300005

Braund, H. y DeLuca, C. (2018). Elementary students as active agents in their learning: an empirical study of the connections between assessment practices and student metacognition. The Australian Association for Research in Education, 45(1), 65-85. https://doi.org/10.1007/s 13384-018-0265-Z

Buck Institute for Education. (2013). What is project-based learning? Recuperado de http://pblonline.org/About/whatisPBL.html

Buscá, F. Pintor, P., Martínez, L. y Peire, T. (2010). Sistemas y procedimientos de evaluación formativa en docencia universitaria: resultados de 34 casos aplicados durante el curso académico 2007-2008. Estudios sobre Educación, 18, 255-276.

Carless, D., Joughin, G. y Mok, M. (2006). Learning-oriented assessment: Principles and practice. Assessment Eं Evaluation in Higher Education, 31(4), 395-398. https://doi.org/10.1080/02602930600679043

Delgado, V., Ausín, V., Hortigüela, D. y Abella, V. (2016). Evaluación entre iguales: Una experiencia de evaluación compartida en Educación Superior. EDUCADI, 1(1), 9-24.

Delgado, V., Hortigüela, D., Ausín, V. y Abella, V. (2018). El Blog como Instrumento de Mejora para la Autorregulación del Aprendizaje del Estudiante Universitario. Estudios Pedagógicos, 44(2), 171-184 https://doi.org/10.4067/S0718-07052018000200171

Durán, D. (2014). Aprenseñar: evidencias e implicaciones educativas de aprender enseñando. Madrid: Narcea.

Finkelstein, N., Hanson T., Huang., C. W., Hirschman, B. y Huang, M. (2010). Effects of problembased economics on high school economics instruction. Washington, DC: US Department of Education.

Fraile, J., Pardo, R. y Panadero, E. (2017). ¿Cómo emplear las rúbricas para implementar una verdadera evaluación formativa? Revista Complutense de Educación, 28(4), 1321-1334. https://doi.org/10.5209/RCED.51915

Grahame, S. D. (2011). Science education in a rapidly changing world. Nueva York, NY: Hauppauge. 
González Losada, S. y Triviño García, M. A. (2018). Las estrategias didácticas en la práctica docente universitaria. Profesorado. Revista de Currículum y Formación del Profesorado, 22(2), 371-378. https://doi.org/10.30827/profesorado.v22i2.7728

Gómez Ruíz, M. y Quesada Serra, V. (2017). Coevaluación o evaluación compartida en el contexto universitario: la percepción del alumnado de primer curso. Revista Iberoamericana De Evaluación Educativa, 1O(2), 9-30. https://doi.org/10.15366/riee2017.10.2.001

Hortigüela, D., Palacios, A. y López-Pastor, V. (2019). The impact of formative and shared or coassessment on the acquisition of transversal competences in higher education. Assessment E Evaluation in Higher Education, 44(6), 933-945. https://doi.org/10.1080/02602938.2018.1530341

Hortigüela, D., Pérez Pueyo, A. Abella García, V. (2015). ¿Cómo influye el sistema de evaluación en la percepción del alumnado? @tic. Revista d'Innovació Educativa, 14, 82-89. https://doi.org/10.7203/attic.14.4170

Hortigüela, D., Pérez-Pueyo, A. y González-Calvo, G. (2019). Pero... ¿A qué nos referimos realmente con la Evaluación Formativa y Compartida?: Confusiones Habituales y Reflexiones Prácticas. Revista Iberoamericana de Evaluación Educativa, 12(1), 13-27. https://doi.org/10.15366/riee2019.12.1.001

Hortigüela, D., Abella, V., Delgado, V. y Ausín, V. (2016). Influencia del sistema de evaluación empleado en la percepción del alumno sobre su aprendizaje y las competencias docentes. Infancia, Educación y Aprendizaje (IEY A), 2(1), 20-42.

Hortigüela, D., Sánchez-Santamaría, J., Pérez-Pueyo, A. y Abella, V. (2019). Social networks to promote motivation and learning in higher education from the students'perspective. Innovations in Education and Teaching International, 56(4), 412-422. https://doi.org/10.1080/14703297.2019.1579665

López-Pastor, V. M. y Palacios, A. (2012). Percepción de los futuros docentes sobre los sistemas de evaluación de sus aprendizajes. Revista Teoría de la Educación: Educación y Cultura en la Sociedad de la Información, 13(3), 317-341.

López-Pastor, V. M. y Pérez-Pueyo, Á. (2017). Evaluación formativa y compartida en Educación: experiencias de éxito en todas las etapas educativas. Recuperado de https://buleria.unileon.es/handle/10612/5999

López-Pastor, V., Pintor, P., Muros, B. y Webb, G. (2013): Formative assessment strategies and their effect on student performance and on student and tutor workload: the results of research projects undertaken in preparation for greater convergence of universities in Spain within the European Higher Education Area (EHEA). Journal of Further and Higher Education, 37(2), 163-180. https://doi.org/10.1080/0309877X.2011.644780

Lucena, I. V. (2016). La aplicación de las TIC y la evaluación por competencias en el grado en derecho. International Journal of Educational Research and Innovation (IJERI), 5, 42-54.

Martínez, L., Castejón, J. y Santos, M. L. (2012). Diferentes percepciones sobre evaluación formativa entre profesorado y alumnado en formación inicial en educación física. REIFOP, $15(4), 57-67$.

Palacios, A. y López-Pastor, V. M. (2012). Percepción de los futuros docentes sobre los sistemas de evaluación de sus aprendizajes. Revista Teoría de la Educación: Educación y Cultura en la Sociedad de la Información, 13(3), 317-341.

Panadero, E. y Alonso-Tapia, J. (2013). Revisión sobre autoevaluación educativa: evidencia empírica de su implementación a través de la autocalificación sin criterios de evaluación, rúbricas y guiones. Revista de Investigación en Educación, 11(2), 172-197. 
Roberts, L. D. y Allen, P. J. (2015). Exploring ethical issues associated with using online surveys in educational research. Educational Research and Evaluation, 21, 95-108. https://doi.org/10.1080/13803611.2015.102442 1

Sanmartí, N. (2007). Evaluar para aprender. 10 ideas clave. Barcelona: Graó.

Thomas, J. W. (2000). A review of research on project-based learning. Los Ángeles, CA: Autodesk Foundation.

Valero-García, M. y de Cerio, L. M. D. (2005). Autoevaluación y co-evaluación: estrategias para facilitar la evaluación continuada. En Actas del Simposio Nacional de Docencia en Informática, SINDI. Recuperado de http://bit.ly/1G1B1xu.

Walker, A. y Leary, A. (2009). Problem based learning Meta-Analysis: Differences across problem types, implementation types, disciplines and assessment levels. The Interdisciplinary Journal of Problem-Based Learning, 3(1), 12-43. https://doi.org/10.7771/1541-5015.1061

\section{Breve $\mathrm{Cv}$ de los autores}

\section{Víctor Abella García}

Profesor Contrado Doctor en el Departamento de Ciencias de la Educación de la Universidad de Burgos. Actualmente es el director del Centro de Enseñanza Virtual de la Universidad de Burgos, encargado de la puesta en marcha y desarrollo de cinco titulaciones virtuales en la modalidad on line. Uno de sus intereses en investigación y futura línea de trabajo es conocer qué factores subyacen a la adopción o no de una tecnología y su implantación en las prácticas pedagógicas. Derivándose de esta línea el otro gran interés que es el diseño de actividades formativas que sean eficaces para el profesorado y que proporcionen modelos pedagógicos de integración de las TIC en el aula para facilitar la transición del profesorado de modelos más tradicionales a metodologías más actuales como por ejemplo el aprendizaje basado en proyectos. ORCID ID: https://orcid.org/0000-0001-9406-9313. Email: vabella@ubu.es

\section{Vanesa Ausín Villaverde}

Profesora en el Departamento de Ciencias de la Educación de la UBU. Imparto docencia en los Grados de Maestro en Educación Primaria, Infantil y Pedagogía, en materias relacionadas de las TIC aplicadas a la educación. Entre las líneas de investigación, se pueden destacar la aplicación de las tecnologías de la información y la comunicación en la formación inicial de los futuros maestros, la evaluación formativa y el uso de metodologías activas para mejorar los procesos de enseñanza y aprendizaje en la universidad. ORCID ID: https://orcid.org/0000-0002-8943-6251. Email: vausin@ubu.es

\section{Vanesa Delgado Benito}

Profesora Contratada Doctor del Área de Didáctica y Organización Escolar de la Universidad de Burgos. Formo parte del Grupo de Investigación EDINTEC y del Grupo de Innovación Docente TICEF. Soy miembro de la Sociedad Española de Pedagogía (SEP) y de la Red Universitaria de Tecnología Educativa (RUTE). Entre mis principales líneas de investigación destaca la formación inicial y permanente del profesorado y las Tecnologías de la Información y la Comunicación en el ámbito educativo. ORCID ID: https://orcid.org/0000-0001-8168-7120.Email: vdelgado@ubu.es 


\section{Raquel Casado Muñoz}

Raquel Casado Muñoz es Maestra, Licenciada en Ciencias de la Educación y Doctora por la Universidad de Burgos, con Premio extraordinario de Doctorado. Docente en la Universidad de Burgos desde 1996, es Profesora Titular del Área de Didáctica y Organización Escolar. Ha dirigido el Instituto de Formación e Innovación Educativa de la misma Universidad (2008/2012), desde donde impulsó diversos planes de formación del profesorado y la creación de grupos de innovación docente. Sus líneas de investigación se orientan hacia la formación del profesorado, la innovación educativa y la promoción de la inclusión educativa. Ha participado como investigadora principal y/o colaboradora en 20 proyectos regionales, nacionales, y europeos. Coordina el Grupo de Investigación Reconocido "Educación, Inclusión y Tecnología" (EDINTEC). ORCID ID: https://orcid.org/0000-0002-9070-6298. Email: rcasado@ubu.es 